\title{
DEDICATION TO ARCADIO POVEDA
}

\author{
Helmut A. Abt ${ }^{1}$ \\ RESUMEN
}

Es apropiado que esta reunión sobre estrellas dobles y múltiples sea en honor a Arcadio Poveda, quien ha encontrado muchos resultados sobre estos sistemas. Este texto menciona algunos de esos resultados, así como otros aspectos de su investigación y de sus logros.

\section{ABSTRACT}

It is appropriate that this meeting about double and multiple stars be dedicated to Arcadio Poveda, who has derived so many results about such systems. This dedication mentions some of those results, as well as some other details of his research and accomplishments.

\section{Key Words: BINARIES: GENERAL - STELLAR DYNAMICS}

Arcadio Poveda has been mainly concerned with problems of dynamics, especially stellar dynamics. He has been particularly effective because he understands the theoretical background but has concentrated primarily on the interpretation of observational data. He reads widely and thinks about problems seemingly unrelated to stars, such as the paper he will give at this meeting concerning the meteoritic impact of 65 million years ago.

Arcadio's thesis at the University of California, Berkeley, was under Shane and Neyman. Shane and Wirtanen were studying the distribution of galaxies in the sky, and finding much clustering. Was that due to a real clustering of galaxies or the result of spotty interstellar absorption? The latter idea had been modeled in a series of papers by Chandrasekhar and Munch. Arcadio solved the problem (Poveda 1957) by counting the distribution of faint stars. He concluded that there was no correlation between the clustering of stars and that of galaxies, so the galaxy clustering is real, not due to absorption.

Next, Arcadio became interested in the mass/luminosity ratio for spherical systems. From data in the literature he found (Poveda 1961) that the relation $\log M / M_{\odot}=0.5-0.55 M_{p g}$ holds all the way from globular clusters to clusters of galaxies. That is a range of over $20 \mathrm{mag}$, and his relation allowed the determination of galactic masses where rotation curves were not available. Work by Oort and Zwicky had already discussed the importance of missing mass in such systems.

Poveda, Cruz, \& Batiz (1960) also explored the nature of the stellar orbits in spherical systems. They used numerical simulations, de Vaucouleurs'

\footnotetext{
${ }^{1}$ Kitt Peak National Observatory, Tucson, AZ, USA.
}

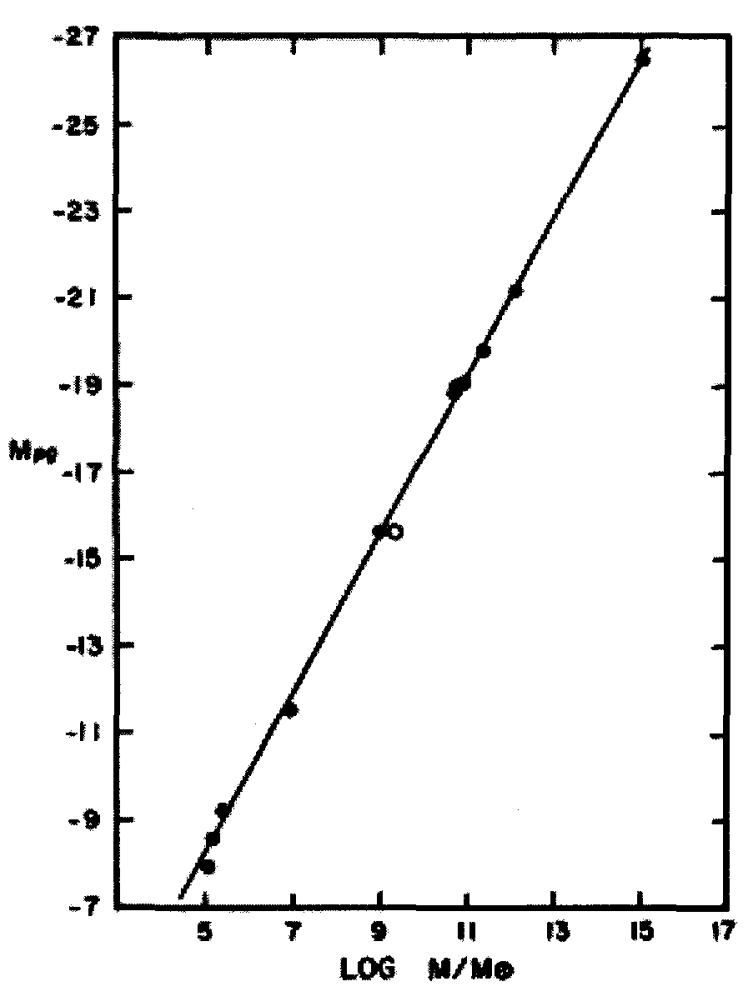

Fig. 1. Poveda found that for spherical systems from globular clusters to clusters of galaxies the relationship between their photographic absolute magnitudes (ordinates) and the logarithms of their masses in solar units (abscissae) is a linear one. (Reprinted from ApJ, 134, 910, 1961.)

brightness distribution, and the known lack of a velocity dispersion variation with radius. They found that most of the orbits were circular, but that about one sixth are radial. 


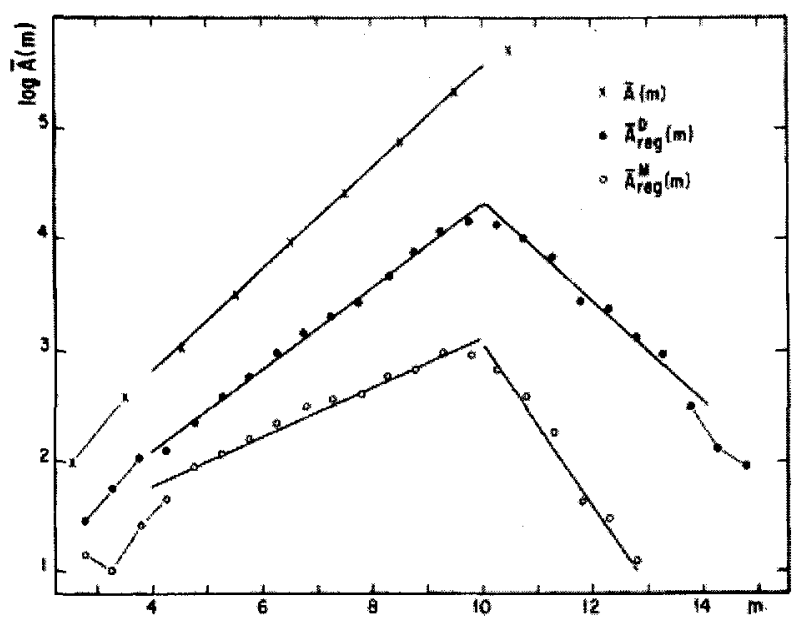

Fig. 2. The number of double stars (middle lines) and multiple stars (bottom lines) are plotted as a function of apparent visual magnitudes, $m$. The top line shows the number of single field stars. The symbols represent the observational data, and the lines are least-squares fits. These show incompleteness starting at magnitude 10. (Reprinted from ApJ, 258, 589, 1982.)

While on sabbatical in Paris, Arcadio became interested in type II supernovae. With Woltjer he discovered a linear relation between the radio brightnesses and apparent diameters of supernova remnants. That relation enabled them to determine distances, which they used to find that type II supernova remnants delineate the Galaxy's spiral arms at least as well as do stellar clusters. They also pointed out for the first time the connection between such remnants and some X-ray sources.

When he was a student in Berkeley, Arcadio was impressed by a colloquium that Oort gave on what is now called the Oort cloud. This led him to wonder about the effect of perturbations on binary orbits. He reasoned that wide binaries should be disrupted. With numerical simulations he and Carlos Cruz-González obtained results that differed significantly from theoretical work by Chandrasekhar and by Ambartsumian (Cruz-González \& Poveda 1971).

A natural follow-up on that work was to wonder about the origin of runaway stars. Zwicky and Blaauw had proposed that they were ejected by supernovae. However, that mechanism was shown to be ineffective unless the supernovae ejected unrealistically large amounts of mass. Instead, with students Christine Allen and Jose Ruiz, Arcadio showed by n-body simulations that perturbations in young collapsing clusters will do the job (Poveda, Ruiz \& Allen 1967).
This let to an interest in Trapezium systems. Parenago (1953) had proposed that all Trapezium systems were expanding and unstable, and derived a surprisingly short lifetime of 10,000 years for them. There was later observational evidence that the Orion Trapezium was expanding. Christine Allen and Arcadio, working with Charles Worley, tested this by using the measurements obtained by visual double-star observers of binary separations in Trapezia. They were often available for intervals up to 150 years. In 42 Trapezium systems they (Allen, Poveda \& Worley 1974) found no evidence for overall expansion, although there was evidence that a few stars were being ejected.

They (Allen \& Poveda 1974) also studied the dynamical lifetime of Trapezia and concluded that it was about 30 crossing times or at least $10^{6} \mathrm{yr}$. In their numerical simulations they found that during collisional interactions there is a tendency for the most massive stars to pair together. This confirmed earlier results by Aarseth \& Hills (1972).

A clever study was to determine the amount of material that is being ejected from the galactic center. Joe Weber had done gravitational-wave measurements that suggested that about $200 M_{\odot}$ of material was being ejected each year. If that continued for much of the lifetime of the Galaxy, the central region would initially have had about $2.4 \times 10^{12} M_{\odot}$. Then the density in the disk would have been much higher initially, and globular clusters would have been destroyed by passing through the disk. Also, wide binaries would have been destroyed in collisions with passing stars. Previously, Poveda \& Allen (1975a) had obtained a more realistic mass for $\omega$ Cen and a galactic orbit for it. Its pericentric distance was only $1.3 \mathrm{kpc}$. It would have been destroyed by the galactic center if the center had had a much higher mass. Poveda \& Allen (1975b) derived a maximum mass loss from the center of only $1 M_{\odot}$ per year.

I will mention one more research project that is pertinent to this Colloquium. Then I will review non-research work that Arcadio has been doing. Poveda, Allen, \& Parrao (1982) studied double and multiple stars in the IDS, the Index Catalog of Double Stars (Jeffers, van den Bos, \& Greeby 1963), and wondered about its completeness. Statistically they found that the catalog was complete to tenth magnitude but then failed to include many physical systems. They devised a " $1 \%$ filter" which meant that unless the probability of finding a field star within the radius of a possible companion was less than $1 \%$, the companion was rejected as probably being an op- 
tical companion. They then concluded that all stars have at least one physical visual eompanion and one third of them have multiple companions.

Now I would like to tell about other things that Arcadio has been doing. When he returned from Berkeley in 1956 with a Ph.D., he became one of only three Mexican astronomers. The others were Haro and Paris Pismis. They started to teach astronomy to physics students, partly to interest them in astronomy. Soon they attracted good students. Now there are about 100 Mexican astronomers, of whom 90 are members of the IAU.

Arcadio has been very active in public education, writing more than 60 popular articles about astronomy, giving more than 10 public lectures, various TV interviews and programs, and writing a book with Miguel Angel Herrera on dark matter, the only one on that topic published in Spanish.

It became obvious that if Mexican astronomers were to do well, they needed and observatory with dark skies. Tacubaya Observatory was enveloped within Mexico City and Tonantzintla Observatory was threatened by a growing Puebla. Under Arcadio's directorship a site survey led to the establishment of San Pedro Mártir Observatory in Baja California, with its 2.1, 1.5, and 0.8-meter telescopes. Also, they built a scientific center in Ensenada for astronomy, instrumentation and oceanography. Arcadio was also involved in setting up an optics center in Leon, Guanajuato, and a University Space Program that has built two satellites. More recently Arcadio has been a member of the Governing Board of the National University, which is equivalent to our boards of trustees. Hey, that's a lot of accomplishments for a young man like Arcadio!

\section{REFERENCES}

Aarseth, S. L., \& Hills, J. G. 1972, A\&A, 21, 255

Allen, C., \& Poveda, A. 1974, IAU Symp. 62, The Stability of the Solar System and Small Stellar Systems, ed. Y. Kozai (Dordrecht: Reidel), 239

Allen, C., Poveda, A., \& Worley, C. E. 1974, Rev. Mex. Astron. Astrofis., 1, 101

Cruz-González, C., \& Poveda, A. 1971, Ap\&SS, 13, 335

Jeffers, H.M., van den Bos, W. H., \& Greeby, F. M. 1963, Publ. Lick Obs., 21

Parenago, P. P., 1953, AJ(USSR), 30, 249

Poveda, A. 1957, Bol. Obs. Tonantzintla y Tacubaya, 15, 9

Poveda, A. 1961, ApJ, 134, 910

Poveda, A., \& Allen, C. 1975a, ApJ, 197, 155

Poveda, A., \& Allen, C. 1975b, ApJ, 200, 42

Poveda, A., Allen, C., \& Parrao, L. 1982, ApJ, 258, 589

Poveda, A., Cruz, C. \& Batiz, G. 1960, AJ, 65, 497

Poveda, A., Ruiz, J, \& Allen, C. 1967, Bol. Obs. Tonantzintla y Tacubaya, 4,86

Helmut A. Abt: Kitt Peak National Observatory, PO Box 26732, Tucson, AZ 85726-6732, USA (abt@noao.edu). 


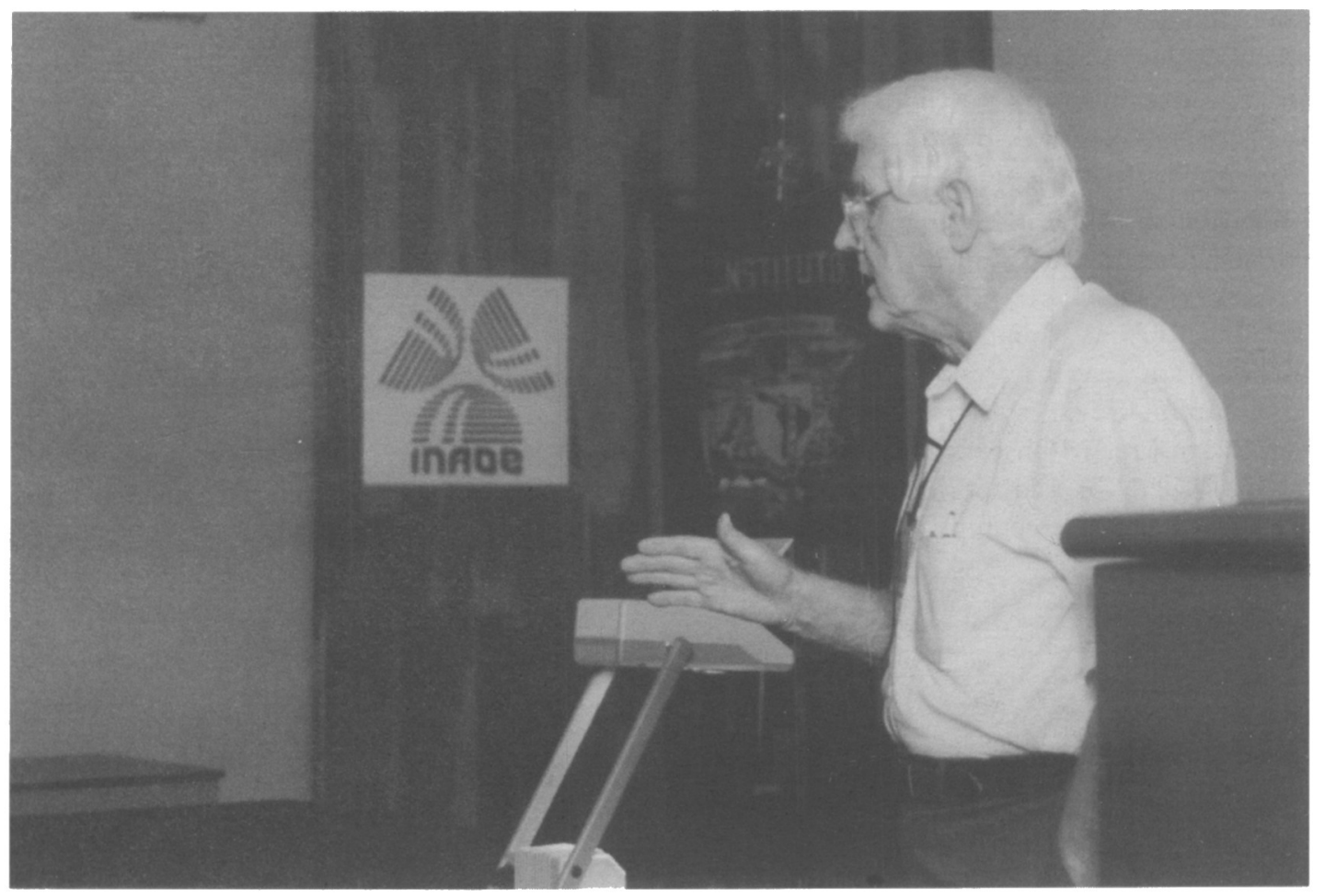

Helmut Abt. 